\title{
Rotation of antimicrobial therapy in the intensive care unit: impact on incidence of ventilator-associated pneumonia caused by antibiotic-resistant Gram-negative bacteria
}

\author{
E. Raineri • L. Crema • S. Dal Zoppo • A. Acquarolo • \\ A. Pan • G. Carnevale • F. Albertario • A. Candiani
}

Received: 1 February 2010 /Accepted: 6 May 2010 /Published online: 4 June 2010

(C) Springer-Verlag 2010

\begin{abstract}
The development of antibiotic resistance is associated with high morbidity and mortality, particularly in the intensive care unit (ICU) setting. We evaluated the effect of an antibiotic rotation programme on the incidence of ventilator-associated pneumonia (VAP) caused by antibiotic-resistant Gram-negative bacteria. We conducted a 2-year before-and-after study at two medical-surgical ICUs at two different tertiary referral hospitals. We included all mechanically ventilated patients admitted for $\geq 48 \mathrm{~h}$ who developed VAP. From 1 January through 31 December 2007, a quarterly rotation of antibiotics (piperacillin/tazobactam, fluoroquinolones, carbapenems and cefepime/ceftazidime) for the empirical treatment of VAP was implemented. We analysed the incidence of VAP and the antibiotic resistance patterns of the responsible patho-
\end{abstract}

E. Raineri $(\bowtie) \cdot$ L. Crema $\cdot$ F. Albertario

Servizio di Rianimazione, Terapia Intensiva e Neuroanestesia, Istituti Ospitalieri di Cremona,

viale Concordia 1 ,

26100 Cremona, Italy

e-mail: rainerineto@libero.it

S. Dal Zoppo

Istituto di Malattie Infettive e Tropicali, Spedali Civili,

Università di Brescia,

piazzale Spedali Civili 1,

25100 Brescia, Italy

\section{A. Acquarolo • A. Candiani}

Istituto di Anestesia e Rianimazione, Spedali Civili,

Università di Brescia,

piazzale Spedali Civili 1,

25100 Brescia, Italy

\section{A. Pan $\cdot$ G. Carnevale}

Divisione di Malattie Infettive, Istituti Ospitalieri di Cremona, viale Concordia 1 ,

26100 Cremona, Italy gens in 2006, before (P1) and, in 2007, after (P2) the introduction of the scheduled rotation programme. Overall, there were 79 VAP episodes in P1 and 44 in P2; the mean incidence of VAP was 20.96 cases per 1,000 days of mechanical ventilation (MV) during P1 and 14.97 in P2, with no significant difference between periods on segmented regression analysis. We observed a non-significant reduction of the number of both the poly-microbial (14 [17.7\%] in P1 and 5 [10.6\%] in $\mathrm{P} 2[p=0.32])$ and of the antibiotic-resistant Gram-negative bacteria-related VAP (42 [45.2\%] in P1 and 16 [34\%] in P2 [ $p=0.21])$. Conversely, the number of VAP caused by Pseudomonas aeruginosa passed from 8.35 per 1,000 days of MV in P1 to 2.33 per 1,000 days of MV in P2 $(p=0.02)$. No difference in ICU mortality and crude in-hospital mortality between P1 and P2 was noted. Moreover, no significant change of microbial flora isolated through clinical cultures was observed. We were able to conclude that, despite global microbial flora not being affected by such a programme, antibiotic therapy rotation may reduce the incidence of VAP caused by antibiotic-resistant Gram-negative bacteria in the ICU, such as Pseudomonas aeruginosa. The application of this programme may also improve antibiotic susceptibility. However, further studies are needed to confirm our results.

\section{Introduction}

The development of antimicrobial resistance is a growing problem worldwide, and infections due to multi-resistant pathogens, both Gram-positive and Gram-negative, have steadily increased over the years [1-3]. Infections with these organisms have been associated with greater mortality $[4,5]$, prolonged hospitalisation [6] and increased costs [7-10].

Multiple strategies have been employed to control the emergence and spread of antibiotic resistance. These 
include colonisation surveillance [2], barrier methods [2, 11-13], antibiotic stewardship [2, 14], decision support $[2,15]$, formulary restriction $[2,16,17]$ and antibiotic cycling or rotation [18-25].

Antibiotic cycling or rotation is based on the scheduled substitution of an antibiotic class with another one having a comparable spectrum of activity but not sharing the same mechanism of resistance. In the ICU setting, cycling has been associated with conflicting results. A limited number of studies has shown a decreased incidence of antibioticresistant Gram-negative infections, particularly ventilatorassociated pneumonia (VAP), a decreased resistance pattern of Pseudomonas aeruginosa and lower infection-related mortality rates [19-21, 24]. Other studies have not observed any advantage of this strategy [26]. To date, the synthesis of these results is that there is insufficient evidence to recommend antibiotic cycling as a standard measure to reduce antibiotic resistance [27, 28].

The purpose of this study was to determine the effectiveness of an antimicrobial rotation programme in reducing the incidence of VAP attributed to antibioticresistant Gram-negative bacteria.

\section{Materials and methods}

\section{Setting}

The study was performed at two different ICUs of two different hospitals in Lombardy, Italy.

Centre A is represented by the medical/surgical ICU of the Istituti Ospitalieri di Cremona, with 10 beds and admitting about 400 patients per year. The ICU is structured as follows: 1 two-bed bays, 2 three-bed bays and 2 single bedrooms. The Istituti Ospitalieri di Cremona is a 600-bed community hospital that serves a population of approximately 150,000 inhabitants and admits about 28,000 patients every year.

Centre B is represented by the $2^{\circ}$ Servizio di Anestesia e Rianimazione of the Spedali Civili di Brescia, it has 10 beds and admits about 400 medical/surgical patients per year. The ICU is structured as follows: 2 four- and 2 onebed bays. The Spedali Civili di Brescia is an 1,800-bed teaching facility that serves a population of approximately $1,000,000$ inhabitants and admits about 80,000 patients every year.

\section{Study design}

We carried out a "before-after" study of 2 years (from 1 January 2006 through 31 December 2007). Inclusion criteria were age $>18$ years, ICU stay $>48 \mathrm{~h}$ and mechanical ventilation (MV) lasting more than $48 \mathrm{~h}$.
The primary outcome of the study was to determine whether the incidence of VAP attributed to antibioticresistant Gram-negative bacteria could be reduced using a scheduled change of antibiotic classes. The secondary outcome was to determine the effectiveness of an antimicrobial rotation programme in reducing the antimicrobial resistance spectrum of antibiotic-resistant Gram-negative bacteria. We chose VAP as the nosocomial parameter to evaluate because it was the most frequent nosocomial infection occurring in both ICUs.

The study was divided into two periods:

- Period 1 (P1), from 1 January 2006 through 31 December 2006, prior to the introduction of the antibiotic rotation programme.

- Period 2 (P2), from 1 January 2007 to 31 December 2007, after the introduction of the antibiotic rotation programme.

\section{Antibiotic prescription}

During P1, the choice of antibiotic prescription for the treatment of VAP was left to the physician's discretion, both for molecule selection and duration of therapy. However, the antibiotics used most were piperacillin/tazobactam and levofloxacin.

During P2 we introduced a protocol based on a quarterly rotation of antibiotics (piperacillin/tazobactam, quinolones, carbapenems and cefepime/ceftazidime) for the treatment of VAP. The new programme was as follows:

- 1 st trimester piperacillin/tazobactam: $4.5 \mathrm{~g}$ intravenously (i.v.), every $6 \mathrm{~h}$.

- 2nd trimester fluoroquinolones: ciprofloxacin $400 \mathrm{mg}$ i. v. b.i.d. or levofloxacin $500 \mathrm{mg}$ b.i.d.

- 3rd trimester carbapenems: imipenem 1 g i.v. g8h or meropenem $1 \mathrm{~g}$ q8h.

- 4th trimester 3rd/4th generation cephalosporins: ceftazidime or cefepime $6 \mathrm{~g}$ /day i.v. continuous infusion.

Standard duration of therapy was 8 days. Unless in the case of resistance, the antibiotic of choice was maintained for the treatment's duration without applying a deescalation strategy when the results from microbiology were available. In cases of different physicians' judgement and when Pseudomonas aeruginosa infection was highly suspected, antibiotic treatment could be prolonged to 14 days. Given the observational nature of this study, informed consent was not required.

\section{Definitions}

For definitions of infection, systemic inflammatory response syndrome, sepsis, severe sepsis and septic 
shock, we referred to the $2001 \mathrm{SCCM} / \mathrm{ESICM} / \mathrm{ACCP} /$ ATS/SIS International Sepsis Definitions Conference [29].

For the definition of VAP we referred to the guidelines of the 2005 American Thoracic Society-Infectious Disease Society of America [30]. VAP was defined as any lower respiratory tract infection that developed after 2 days of MV. Clinical suspicion of VAP was defined as a new, progressive, or persistent $(>24 \mathrm{~h}$ ) infiltrate on the chest radiograph, with two or more of the following criteria:

1. Fever $>38.3{ }^{\circ} \mathrm{C}$ or hypothermia $<36^{\circ} \mathrm{C}$

2. Purulent endotracheal aspirate

3. Leukocytes count $>10,000 / \mathrm{mm}^{3}$ or $<4,000 / \mathrm{mm}^{3}$

Every patient suspected of having pneumonia underwent, within $24 \mathrm{~h}$, lower respiratory tract microbiological sampling, always before initiating empirical antimicrobial treatment. To establish a microbiological diagnosis we performed, whenever feasible, a broncho-alveolar lavage (BAL); as an alternative method, when bronchoscopy was not immediately available, we used a blind sampling technique (blind mini-BAL) whose diagnostic accuracy has been widely established $[31,32]$. A case of VAP was defined as microbiologically confirmed when bacteria were isolated in significant quantities from BAL samples $\left(\geq 10^{4} \mathrm{CFU} / \mathrm{ml}\right)$. We defined "early VAP" as those occurring during the first 5 days of MV and "late VAP" as those occurring after 5 days of MV.

For definitions of infections, we referred to the International Sepsis Forum Consensus Conference on Definitions of Infections in the Intensive Care Unit [33]. The organism associated with infection was defined by the isolation of the germ in a biological material in the presence of signs and symptoms of infection.

\section{Micro-organism susceptibility}

Antibiotic-resistant Gram-negative bacteria were defined as germs being resistant to at least one class of antibiotics typically used in the treatment of Gram-negative bacterial infections. We defined as multi-drug resistant (MDR) a germ that was resistant to at least three antibiotic classes. We analysed data regarding resistant Pseudomonas aeruginosa, Stenotrophomonas maltophilia, Acinetobacter baumanii and extended spectrum $\beta$-lactamase (ESBL) Enterobacteriaceae. The antibiotics we considered for resistance were: aminoglycosides (gentamycin, tobramycin, amikacin), third/fourth generation cephalosporins (ceftazidime/cefepime), extended-spectrum penicillins (piperacillin/tazobactam), fluoroquinolones (ciprofloxacin, levofloxacin) and carbapenems (imipenem/meropenem).
Compliance with the rotation protocol

For each patient we evaluated if the prescribed therapy was in accordance or not with the rotation protocol. We defined three categories:

1. Exact antibiotic class prescribed

2. Acceptable deviation, e.g., allergy, drug interaction, antibiotic-resistant germ

3. Major deviation

We considered categories 1 and 2 as adhering to the rotation protocol.

Antibiotic utilisation

Although analysis of antibiotic consumption was not an objective of this study; we reported the frequency of use of commonly prescribed antibiotic classes for the treatment of VAP before and after the introduction of rotation, expressed as the number of antibiotic cycles per 1,000 patient days.

According to the Anatomical, Therapeutic, Chemical (ATC) classification system with Defined Daily Doses (DDDs), the amount in grams for an antimicrobial agent was converted into a number of DDDs [34]. The consumption density for each antibiotic class was defined as the division of the number of DDDs by the number of patientdays.

\section{Infection control measures}

All the standard infection control measures were maintained active and were not modified during the two periods. All patients were placed, whenever possible, in a $30-45^{\circ}$ (semi-recumbent) position. Health care workers were invited to wash their hands with chlorhexidine-based soap or rub hands with alcohol-based solutions before and after patient contact. In the case of colonisation or infection caused by a multi-resistant germ, either Gram-positive or Gram-negative, standard contact precautions, including hand hygiene and use of gloves, gowns, masks and caps were applied during all medical and nursing procedures. Those patients were isolated in single rooms or cohorted, depending on bed availability [35, 36]. No specific interventions to modify infection control measures were performed in the two units over the study period.

Data collection and validation

Data were collected by ICU medical staff members: ER and LC at Centre A, and ER and SD at Centre B. Data were registered in a dedicated database. For each patient we recorded: age, sex, admission diagnosis, Glasgow Coma Scale (GCS), Simplified Acute Physiology Score (SAPS) 2 
and 3 , isolated germs, susceptibility pattern, type, dose and length of antibiotic therapy, days of MV, days of ICU stay and crude ICU mortality. Microbiological results were obtained from the Microbiology Laboratory computerised system of each hospital. All copy-isolates were excluded; only the first positive culture of each germ for each patient was included. Data on antibiotic use were obtained from the Hospital Pharmacy.

Statistical analysis

Continuous variables with normal distribution were expressed as mean \pm standard deviation (SD); those with a non-normal distribution (Shapiro-Wilk W test) were expressed as median values and interquartile range (25th75 th percentile). To compare the categorical variables, we used the $\chi^{2}$ test or Fisher's exact test when necessary. To compare continuous variables the $t$ test or the KruskalWallis test was used, as appropriate. Relative risks (RRs) and their $95 \%$ confidence intervals (CI) were calculated using standard methods.

The SAPS2 was used during 2006 and SAPS3 during 2007. These two parameters have been compared by calculating the predicted in-hospital mortality associated with each SAPS value.

The incidence rate of VAP was expressed as the number of cases per 1,000 patient days and per 1,000 days of MV.

The analysis of rate differences between periods was performed utilising the segmented regression analysis, a powerful method for estimating intervention effect in interrupted time series studies [37]. In this study we have an intervention determining two segments, namely: P1 and P2. Each segment is divided into periods of 1 month. Two parameters defined each segment: level and trend. The level is the value of the series at the beginning of a given time interval and the trend is the slope of a given segment. A change in level constitutes an abrupt intervention effect; a change in trend is defined by an increase or decrease in the slope of the segment after the intervention compared with the segment preceding the intervention itself, i.e., represents a gradual change in infection rate during the segment. We fitted the following model: $Y=\beta_{0}+\beta_{1} x$ time $+\beta_{2} x$ interention $1+\beta_{3} x$ timeafterintervention $1+e$ where $\beta_{0}$ represents the intercept, $\beta_{1}$ the baseline time trend, $\beta_{2}$ the level change after the introduction of the rotation protocol, $\beta_{3}$ the trend change after the introduction of the rotation protocol and $e$ the error term. Ordinary least squares regression analysis assumes that error terms associated with each observation are uncorrelated. As time is a predictor in segmented regression analysis, error terms of consecutive observations could be correlated (first order autocorrelation): that is why we applied the Durbin-Watson statistics (values close to 2.00 indicate no serious autocorrelation) and visually inspect a plot of residuals against time.

All the statistical tests were 2-tailed and were considered significant when $P<0.05$. The statistical analysis was conducted using STATA software (Stata Statistical Software, release 8.0, 2003; StataCorp, College Station, TX, USA).

\section{Results}

General population

From 1 January 2006 to 31 December 2007 a total of 1,649 patients were admitted, 827 and 822 during P1 and P2 respectively. Mean age was $58.99 \pm 20.11$ and $61.24 \pm$ 18.76 years in $\mathrm{P} 1$ and $\mathrm{P} 2$ respectively $(p=0.05)$. There were a total of 10,553 patient days; mean length of ICU stay (median; 25th-75th percentile) was $6.88(3 ; 1-9)$ days in P1 and $5.91(2 ; 1-7)$ days in P2 $(p=0.006)$. The total number of days on mechanical ventilation were 6,839 ; the mean duration of mechanical ventilation (median; 25th75th percentile) was $5.57(2 ; 1-7)$ days in P1 and 4.43 (1; $1-5)$ days in $\mathrm{P} 2(p=0.023)$.

Expected in-hospital mortality at 28 days calculated on the basis of the SAPS (SAPS2 during 2006 and SAPS3 during 2007) was $32.75 \%$ in $\mathrm{P} 1$ and $44.11 \%$ in $\mathrm{P} 2$ ( $p<$ $0.0001)$. Median GCS (25th-75th percentile) was $11(7-15)$ in P1 and $11(7-15)$ in P2 $(p>0.99)$.

\section{Patients with VAP}

We enrolled 112 patients with VAP; their baseline characteristics are summarised in Table 1. Epidemiological features were similar in both periods. In-hospital mortality predicted by SAPS was $34.08 \%$ in $\mathrm{P} 1$ and $45.13 \%$ in $\mathrm{P} 2$ $(p=0.03)$, and the crude ICU mortality was similar in both periods (Table 2). Among the pathologies on admission, we observed a reduction in patients with trauma $(44.1 \%$ in P1 vs $29.5 \%$ in $\mathrm{P} 2 ; p=0.12$ ), and an increase in patients with infection ( $7.4 \%$ in $\mathrm{P} 1$ vs $25 \%$ in $\mathrm{P} 2 ; p=0.004)$.

There were a total of 79 VAP episodes in P1 and 44 in P2 (Table 2); the proportion of late onset VAP was similar in the two periods: $57 \%$ in $\mathrm{P} 1$ and $56.8 \%$ in $\mathrm{P} 2(p=0.88)$. The mean incidence rate of VAP was 13.94 episodes per 1,000 patient days (20.96 episodes per 1,000 days of MV) in $\mathrm{P} 1$ and 9.05 episodes per 1,000 patient days (14.97 episodes per 1,000 days of MV) in P2 (Fig. 1). Segmented regression analysis showed no significant difference between periods. 
Table 1 Baseline characteristics of the study cohort

P1: 1 January 2006 to 31 December 2006; P2: 1 January 2007 to 31 December 2007

The Simplified Acute Physiology Score (SAPS) 2 was used during 2006 and SAPS3 during 2007. These two parameters have been compared by calculating the predicted in-hospital mortality associated with each SAPS value

NA, not applicable

\begin{tabular}{llll}
\hline & $\mathrm{P} 1(n=68)$ & $\mathrm{P} 2(n=44)$ & $p$ \\
\hline Male sex, $n(\%)$ & $44(65)$ & $27(69)$ & 0.64 \\
Age, years & $52.26 \pm 20.86$ & $52.7 \pm 18.1$ & 0.92 \\
SAPS2 & $43.24 \pm 14.93$ & $\mathrm{NA}$ & $\mathrm{NA}$ \\
SAPS3 & $\mathrm{NA}$ & $64.44 \pm 11.91$ & $\mathrm{NA}$ \\
Predicted in-hospital mortality (\%) & 34.08 & 45.13 & 0.03 \\
GCS median (25th-75th percentile) & $8(5-11)$ & $8(6-11)$ & 0.62 \\
Surgical state, $n(\%)$ & & & \\
Medical & $37(54.4)$ & $22(50)$ & 0.65 \\
Elective surgery & $2(2.9)$ & $2(4.5)$ & 0.64 \\
Emergency surgery & $29(42.6)$ & $20(45.4)$ & 0.77 \\
Admission pathology, $n(\%)$ & & & \\
Trauma & $30(44.1)$ & $13(29.5)$ & 0.12 \\
Neurological & $27(39.7)$ & $25(56.8)$ & 0.08 \\
Respiratory & $3(4.4)$ & $2(4.5)$ & 0.99 \\
Gastroenteric & $2(2.9)$ & $2(4.5)$ & 0.64 \\
Cardiovascular & $5(7.4)$ & $2(4.5)$ & 0.7 \\
Other & $1(1.5)$ & $2(4.5)$ & 0.56 \\
\hline
\end{tabular}

\section{Microbiology}

\section{Ventilator-associated pneumonia}

There were 79 microbiologically documented cases of VAP (100\%) in P1 and $41(91 \%)$ in P2. A total of 149 micro-organisms were isolated: 93 in $\mathrm{P} 1$ and 47 in $\mathrm{P} 2$ (Table 3). Poly-microbial VAP was diagnosed in 14 cases $(17.7 \%)$ during $\mathrm{P} 1$ and in $5(10.6 \%)$ in $\mathrm{P} 2(p=0.32)$. There were 42 episodes of VAP caused by antibiotic resistant Gram-negative bacteria (45.2\%) in P1 and 16 (34\%) in P2 $(p=0.21)$.

The number of VAP caused by Pseudomonas aeruginosa passed from 8.35 per 1,000 days of MV in P1 to 2.33 per 1,000 days of MV in P2 $(p=0.02)$. We used segmented regression to analyse VAP episodes caused by Pseudomonas aeruginosa per 1,000 days of MV (Fig. 1). We observed:

1. A significant baseline trend $\left(\beta_{1}: 0.64 ; \mathrm{IC}_{95}\right.$ 0.005-1.28; $p=0.048$ )

2. A significant rate reduction after the introduction of the rotation programme $\left(\beta_{2}:-7.54 ; \mathrm{IC}_{95}\right.$ from $-13,81$ to $-1.27 ; p=0.02$ )

3. A significant trend change after the introduction of the rotation programme $\left(\beta_{3}:-1.07 ; \mathrm{IC}_{95}\right.$ from -1.98 to $-0.17 ; p=0.02$ )

Both Durbin-Watson statistics and visual inspection of the plotting of residuals against time showed an absence of autocorrelation.
Table 2 Main clinical outcomes of the study cohort

VAP, ventilator-associated pneumonia; $n$, number of patients with VAP

${ }^{\mathrm{a}}$ The number of VAP episodes is different from the number of patients with VAP because some patients had more than one VAP episode

\begin{tabular}{lllc}
\hline & $\mathrm{P} 1(n=68)$ & $\mathrm{P} 2(n=44)$ & $p$ \\
\hline ICU length of stay, days & $22.42 \pm 16.47$ & $23.04 \pm 15.38$ & 0.88 \\
ICU mortality, $n(\%)$ & $9(17)$ & $10(23)$ & 0.48 \\
In-hospital mortality, $n(\%)$ & $10(19)$ & $12(27)$ & 0.46 \\
Duration of mechanical ventilation, days & $18.21 \pm 16.58$ & $17.02 \pm 14.15$ & 0.66 \\
Infection severity, $n$ (\%) & & & \\
Infection without SIRS & $16(23.5)$ & $22(50)$ & 0.004 \\
Sepsis & $32(47.1)$ & $14(31.8)$ & 0.12 \\
Severe sepsis & $12(17.6)$ & $2(4.5)$ & 0.045 \\
Septic shock & $8(11.8)$ & $6(13.6)$ & 0.77 \\
Total VAP episodes, $n^{\mathrm{a}}$ & 79 & 45 & $\mathrm{NA}$ \\
Episodes of late VAP (\%) & $45(57)$ & $25(56.8)$ & 0.88 \\
Poly-microbial VAP (\%) & $14(17.7)$ & $5(10.6)$ & 0.32 \\
\hline
\end{tabular}


P1

P2

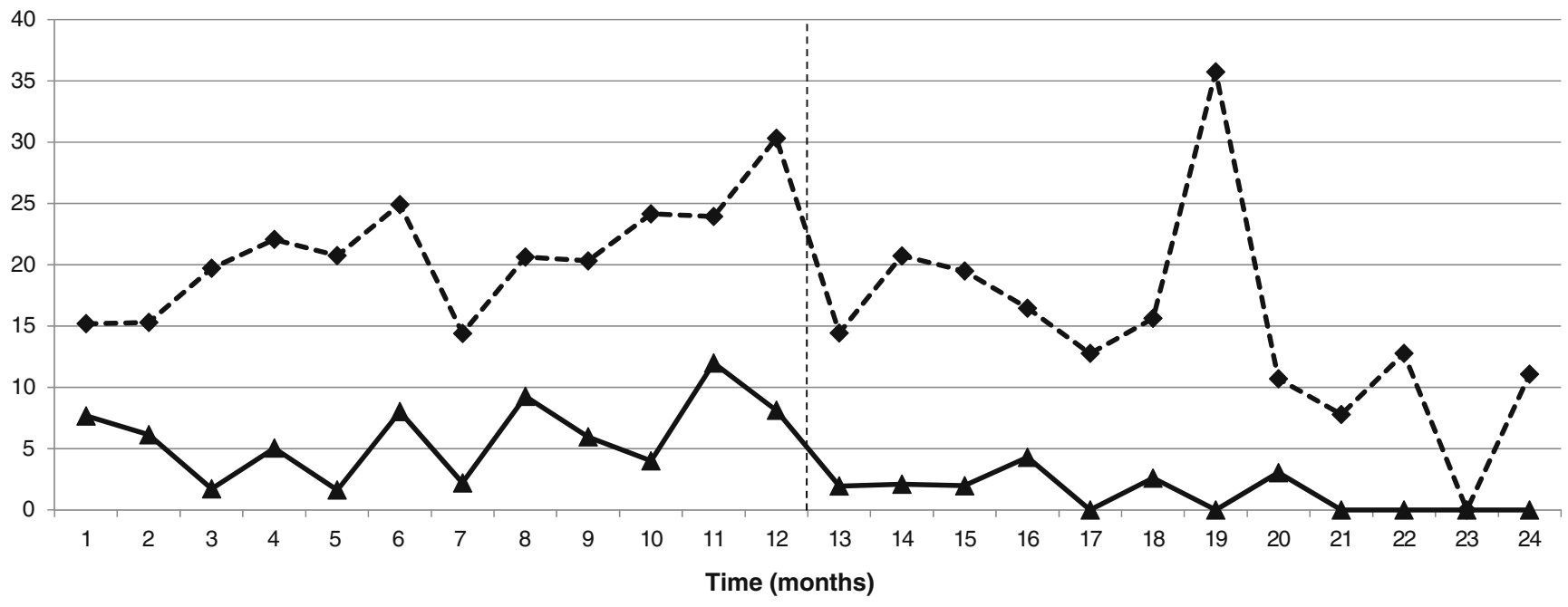

- Number of VAP per 1000 days of mechanical ventilation $\longrightarrow$ Number of VAP caused by P. aeruginosa per 1000 days of mechanical ventilation

Fig. 1 Incidence of overall VAP episodes (dashed line) and VAP episodes caused by Pseudomonas aeruginosa (solid line) per 1,000 days of mechanical ventilation

\section{Microbial flora}

Routine surveillance cultures were not performed. A total of 420 micro-organisms associated with infection were isolated through clinical cultures during the study (data not shown). The proportion of isolates for any single species was similar during the two study periods.

\section{Bacterial resistance}

Percentages of resistance to antimicrobial agents of potentially resistant Gram-negative bacteria responsible for VAP are summarised in Table 4. We observed a reduction in the percentage of Pseudomonas aeruginosa resistant to cefepime $(p=0.05)$ and to aminoglycosides $(p=0.2)$. For Klebsiella spp., Enterobacter spp., Serratia spp. and E. coli, we observed a reduction in resistance toward cefazolin $(p=0.004)$.

Antibiotic utilisation and compliance with rotation protocol

During the study there was a significant reduction in aminoglycoside use for the treatment of VAP: 7.73 vs 2.88 antibiotic courses per 1,000 patient days in P1 and P2 respectively $(p=0.014)$. No differences were observed for the other classes. Concerning the overall antibiotic use (Table 5), we observed a significant increase in the use of extended-spectrum penicillins, carbapenems and metronidazole. Conversely, there was a significant reduction in the use of third/fourth generation cephalosporins, fluoroquinolones, glycopeptides, aminoglycosides and macrolides. Quarterly consumption density of the most frequently prescribed antimicrobial groups in the two ICUs during 2007 is summarised in Fig. 2.

Compliance with rotation protocol was high and similar in both ICUs. Adherence to the rotation protocol was $88 \%$ in centre $\mathrm{A}$ and $83 \%$ in centre $\mathrm{B}$.

\section{Discussion}

This study analyses the effectiveness of an antibiotic rotation programme to reduce the incidence of VAP caused by antibiotic-resistant Gram-negative bacteria in the ICU. We observed a reduction in the VAP rate caused by Pseudomonas aeruginosa per 1,000 days of MV, although there was not an overall reduction in VAP episodes. There was also an augmented susceptibility of Pseudomonas aeruginosa toward cefepime and a reduced frequency of aminoglycosides use, an antibiotic class that is not considered nowadays to be first/second line treatment for VAP. Antibiotic use significantly changed during the study: we observed a significant increase in the use of extendedspectrum penicillins and carbapenems and a significant reduction in the use of third/fourth generation cephalosporins, fluoroquinolones, glycopeptides and aminoglycosides.

Much attention has been focussed in the past on the scheduled rotation of antibiotic therapy as a potential means of limiting the spread of resistant pathogens, but to date it has not been extensively studied to allow definitive conclusions. Although methodological differences make previous studies difficult to compare, a proportion of them reported positive results in terms of decreased incidence of antibiotic-resistant Gram-negative infections, particularly 
Table 3 Micro-organisms associated with VAP
The numbers represent etiological agents of VAP. Some VAP episodes are poly-microbial. All copy-isolates were excluded, only the first positive culture of each germ for each patient was included

\begin{tabular}{lccc}
\hline & $\mathrm{P} 1(n=93)$ & $\mathrm{P} 2(n=47)$ & $p$ \\
\hline Gram-positive, $n$ (\%) & $28(30.1)$ & $13(27.7)$ & 0.76 \\
Staphylococcus aureus & $22(23.7)$ & $11(23.4)$ & 0.97 \\
$\quad$ of which MRSA & $3(3.2)$ & $4(8.5)$ & 0.22 \\
Staphylococcus epidermidis & $0(0)$ & $0(0)$ & $\mathrm{NA}$ \\
Enterococcus spp. & $1(1.1)$ & $0(0)$ & 0.99 \\
Streptococcus pneumoniae & $5(5.4)$ & $2(4.3)$ & 0.99 \\
Other & $0(0)$ & $0(0)$ & $\mathrm{NA}$ \\
Gram-negative, $n$ (\%) & $65(69.9)$ & $34(72.3)$ & 0.76 \\
Escherichia coli & $5(5.4)$ & $4(8.5)$ & 0.48 \\
Pseudomonas aeruginosa & $32(34.4)$ & $7(14.9)$ & 0.016 \\
Haemophylus influenzae & $3(3.2)$ & $6(12.8)$ & 0.06 \\
Klebsiella spp. & $5(5.4)$ & $4(8.5)$ & 0.48 \\
Enterobacter spp. & $3(3.2)$ & $0(0)$ & 0.55 \\
Stenotrophomonas maltophilia & $10(10.8)$ & $5(10.6)$ & 0.98 \\
Serratia spp. & $5(5.4)$ & $3(6.4)$ & 0.99 \\
Proteus spp. & $1(1.1)$ & $1(2.1)$ & 0.99 \\
Citrobacter spp. & $1(1.1)$ & $3(6.4)$ & 0.99 \\
Other & $0(0)$ & & 0.036 \\
\hline
\end{tabular}

VAP [19, 20, 24], infections caused by multi-resistant pathogens [19-21, 24] and infection-attributable mortality [21]. Gruson et al. [19] demonstrated that antimicrobial cycling in a medical ICU, significantly reduced VAP incidence and increased antimicrobial susceptibility, partic- ularly of Pseudomonas aeruginosa, Burkholderia cepacia and methicillin-resistant Staphylococcus aureus (MRSA); these results have also been confirmed in the long term [20]. In a study by Kollef et al. [24] a change in empirical therapy for Gram-negative infection from ceftazidime to

Table 4 Number (\%) of antibiotic-resistant micro-organisms among the principal Gram-negative bacteria responsible for VAP

\begin{tabular}{|c|c|c|c|c|c|c|}
\hline \multirow[b]{2}{*}{ Antibiotic, $n(\%)$} & \multicolumn{2}{|c|}{ P. aeruginosa } & \multicolumn{2}{|c|}{ S. maltophilia } & \multicolumn{2}{|c|}{ KES. + E. coli } \\
\hline & $\mathrm{P} 1(n=32)$ & $\mathrm{P} 2(n=7)$ & $\mathrm{P} 1(n=10)$ & $\mathrm{P} 2(n=5)$ & $\mathrm{P} 1(n=18)$ & $\mathrm{P} 2(n=11)$ \\
\hline Amikacin & $14(43.7)^{*}$ & $1(14.3)^{*}$ & NT & NT & $0(0)$ & $0(0)$ \\
\hline Ampicillin & $32(100)$ & $7(100)$ & NT & NT & $12(66.7)$ & $7(63.6)$ \\
\hline Ampicillin/sulbactam & $32(100)$ & $7(100)$ & NT & NT & $7(38.9)^{*}$ & $2(18.1)$ \\
\hline Aztreonam & $18(56.2)$ & $2(28.6)$ & NT & NT & $4(22.2)$ & $0(0)$ \\
\hline Cefazolin & $32(100)$ & $7(100)$ & NT & NT & $10(55.5)^{* * *}$ & $0(0)^{* * *}$ \\
\hline Cefepime & $18(56.2)^{* *}$ & $1(14.3)^{* *}$ & $3(30)$ & $1(20)$ & $0(0)$ & $0(0)$ \\
\hline Ceftazidime & $18(56.2)$ & $2(28.6)$ & NT & NT & $3(16.7)$ & $0(0)$ \\
\hline Ceftriaxone & $32(100)$ & $7(100)$ & $6(60)$ & $3(60)$ & $4(22.2)$ & $0(0)$ \\
\hline Ciprofloxacin & $12(37.5)$ & $1(14.3)$ & $7(70)$ & $2(40)$ & $0(0)$ & $2(18.1)$ \\
\hline Gentamicin & $14(43.7)^{*}$ & $1(14.3)^{*}$ & NT & NT & $0(0)$ & $0(0)$ \\
\hline Imipenem & $12(37.5)$ & $2(28.6)$ & NT & NT & $0(0)$ & $0(0)$ \\
\hline Levofloxacin & $12(37.5)$ & $1(14.3)$ & NT & NT & $0(0)$ & $2(18.1)$ \\
\hline Piperacillin/tazobactam & $4(12.5)$ & $1(14.3)$ & NT & NT & $3(16.7)$ & $1(9)$ \\
\hline Tobramycin & $14(43.7)^{*}$ & $1(14.3)^{*}$ & NT & NT & $0(0)$ & $0(0)$ \\
\hline Trimethoprim/Sulfamethoxazole & $32(100)$ & $7(100)$ & $0(0)$ & $0(0)$ & $1(0)$ & $1(9)$ \\
\hline
\end{tabular}

KES, Klebsiella spp., Enterobacter spp., Serratia spp.; NT, not tested

${ }^{*} p=0.2$

$* * p=0.05$

$* * * p=0.004$ 
Table 5 Consumption density of the most frequently prescribed antimicrobial groups in the two ICUs during the study period

The consumption density for each antibiotic class was defined as the proportion between the number of DDDs and the number of patient-days

"Other" antibiotics include: trimethoprim/sulphamethoxazole, clindamycin, tigecycline, linezolid, daptomycin and colistin DDD, defined daily dose

\begin{tabular}{lrrcc}
\hline & 2006 & 2007 & RR $(95 \% \mathrm{CI})$ & $p$ \\
\hline Extended-spectrum penicillins & 533.78 & 801.63 & $0.75(0.73-0.78)$ & $<0.0001$ \\
Anti-Pseudomonal penicillins & 224.79 & 212.66 & $1.02(0.98-1.06)$ & 0.27 \\
Third/fourth generation cephalosporins & 235.89 & 148.31 & $1.22(1.18-1.26)$ & $<0.0001$ \\
Carbapenems & 174.28 & 279.18 & $0.76(0.73-0.8)$ & $<0.0001$ \\
Glycopeptides & 240.16 & 166.22 & $1.18(1.14-1.22)$ & $<0.0001$ \\
Fluoroquinolones & 544.21 & 421.37 & $1.14(1.11-1.17)$ & $<0.0001$ \\
Aminoglycosides & 273.66 & 155.97 & $1.17(1.23-1.31)$ & $<0.0001$ \\
Macrolides & 45.17 & 13.38 & $1.48(1.39-1.56)$ & $<0.0001$ \\
Metronidazole & 83.38 & 87.62 & $0.83(0.77-0.88)$ & $<0.0001$ \\
Other & 77.64 & 118.62 & $0.79(0.74-0.85)$ & $<0.0001$ \\
Total DDD per 1000 patient days & $2,432.96$ & $2,404.96$ & & NA \\
\hline
\end{tabular}

ciprofloxacin resulted in a decline in antibiotic-resistant Gram-negative VAP and overall VAP rates without a significant change in BSI incidence or mortality. Raymond et al. [21] studied the impact of rotating empirical antimicrobial regimens among surgical patients during a 2 -year period, demonstrating a significant reduction in multi-drug resistant bacterial infections (both Gram-positive and Gram-negative) and infection-related mortality. In a study by Bennet et al. the implementation of an antibiotic rotation protocol in a surgical ICU resulted in overall improvement in the antibiotic susceptibility profile of Gram-negative microorganisms [38]. Despite these positive results, these studies, as highlighted by Nijssen et al., have important design limitations that increase the risk of confounders such as sub-optimal study design and end point, missing data regarding determination of the germs, different acquisition routes or data regarding infection control practices, such as adherence to hand hygiene [39]. Two different reviews $[28,40]$ emphasised the poor quality of most clinical trials available, concluding that cycling should not be implemented as a routine means of optimising antibiotic prescription and reducing antibiotic resistance. Similar indications are reported by the 2007 Infectious Disease Society of America (IDSA) guidelines on antibiotic stewardship: there have not been enough data in favour of antibiotic cycling to date to recommend this practice [27]. Contrariwise, another review [41] concluded that, although further study is needed, cycling could be an

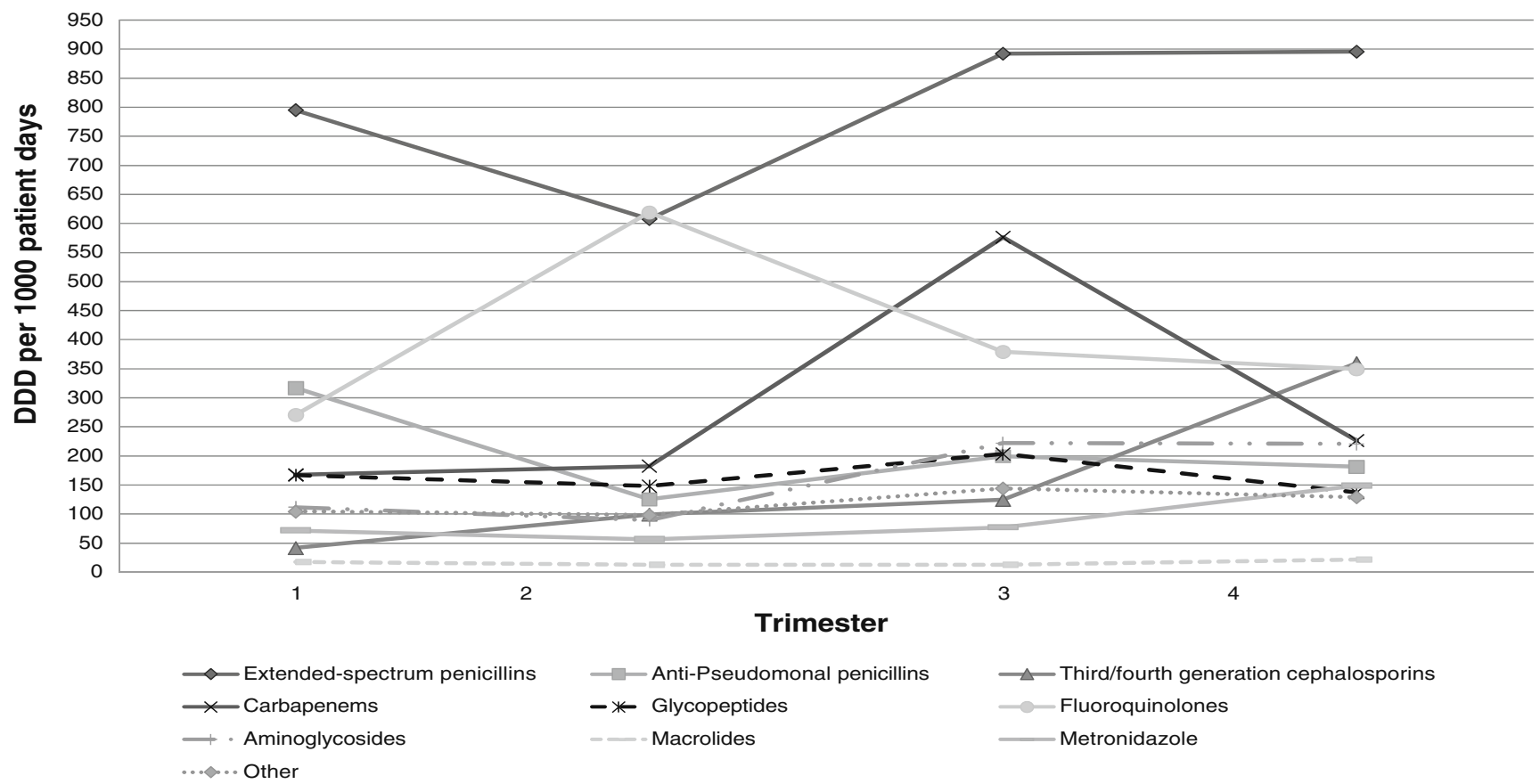

NOTE. The consumption density for each antibiotic class was defined as the proportion between the number of DDDs and the number of patientdays. "Other" antibiotics include: trimetoprim/sulfimethoxazole, clindamycin, tigecyclin, linezolid, daptomycin and colistin.

Fig. 2 Consumption density per quarter of the most frequently prescribed antimicrobial groups in the two ICUs during 2007 
option for controlling antibiotic-resistant Gram-negative infections.

During this study we did not observe any significant reduction in:

\section{Overall VAP incidence \\ 2. Mean duration of ICU stay \\ 3. Mean duration of MV \\ 4. ICU mortality}

Moreover, no significant change in microbial flora isolated through clinical cultures was observed. Interestingly, the rate of VAP caused by Pseudomonas aeruginosa, which represents the most common pathogen, was significantly reduced from $\mathrm{P} 1$ ( 8.35 per 1,000 days of MV) to P2 (2.33 per 1,000 days of $\mathrm{MV} ; p=0.02$ ). We may only suppose that this reduction could be related to the introduction of the rotation programme, since, during the study, all the principal infection control measures were similar and no new interventions had been introduced, although we cannot exclude a "surveillance effect."

We observed a reduced Pseudomonas aeruginosa resistance rate toward cefepime $(p=0.05)$. This could be explained by a low utilisation of the drug in both centres; it is well known, in fact, the correlation between antibiotic use and development of bacterial resistance [42-44]. The change in resistance to cefazolin by KES. and E. coli should be explained by the very infrequent use of this antibiotic in both ICUs during the study. We have no evidence of clonal spread during the first period, although we cannot exclude it. No molecular investigation was performed to identify clonal spreading of these germs. We reported a decreased utilisation of aminoglycosides in P2 $(p=0.014)$. This could be attributed to the exclusion of this drug from the rotation programme and also to an increase in mono-therapy compared with association therapy for the empiric treatment of VAP. Regarding the overall antibiotic use, we found a reorganisation of the antibiotic prescription behaviour in both centres. Third/fourth generation cephalosporins and fluoroquinolones were partly substituted by extended-spectrum and anti-pseudomonal penicillins or carbapenems. Aminoglycosides were rarely used as firstline empiric treatment or prophylaxis; macrolides use was limited to the treatment of community-acquired pneumonia; glycopeptides were mainly prescribed for the treatment of microbiologically documented infections caused by methicillin-resistant Gram-positive cocci.

The main limitation of this study is represented by the small study population, determined mainly by the low VAP incidence observed in both centres, compared with that published in other reports [19, 24], possibly related to the baseline infection control measures, which were already active at the beginning of the study. Second, as VAP was the only infection studied, we cannot estimate the effect of antibiotic rotation on other infections such as bloodstream infection, peritonitis or urinary tract infection. Third, we did not perform routine surveillance cultures in order to evaluate colonisation with antibiotic-resistant germs nor did we perform isolates genotyping in order to define crosstransmission, endogenous selection of resistance or resistance development. Fourth, we did not analyse the costs of antibiotic prescription. Finally, as suggested by McGowan et al. [45], the "before-after" design did not represent the ideal model for the study of cycling.

We were able to conclude that, despite global microbial flora not being affected by such a programme, antibiotic therapy rotation may reduce the incidence of VAP caused by antibiotic-resistant Gram-negative bacteria in the ICU, such as Pseudomonas aeruginosa. The application of this programme may also improve antibiotic susceptibility. However, further studies are needed to confirm our results.

\section{References}

1. Fridkin SK, Gaynes RP (1999) Antimicrobial resistance in intensive care units. Clin Chest Med 20:303-316, viii

2. Kollef MH, Fraser VJ (2001) Antibiotic resistance in the intensive care unit. Ann Intern Med 134:298-314

3 . Vincent JL (2003) Nosocomial infections in adult intensive-care units. Lancet 361:2068-2077

4. Fagon JY, Chastre J, Hance AJ, Montravers P, Novara A, Gibert C (1993) Nosocomial pneumonia in ventilated patients: a cohort study evaluating attributable mortality and hospital stay. Am J Med 94:281-288

5. Kollef MH, Silver P, Murphy DM, Trovillion E (1995) The effect of late-onset ventilator-associated pneumonia in determining patient mortality. Chest 108:1655-1662

6. Goldmann DA, Weinstein RA, Wenzel RP, Tablan OC, Duma RJ, Gaynes RP et al (1996) Strategies to prevent and control the emergence and spread of antimicrobial-resistant microorganisms in hospitals. A challenge to hospital leadership. JAMA 275:234 240

7. Carmeli Y, Troillet N, Karchmer AW, Samore MH (1999) Health and economic outcomes of antibiotic resistance in Pseudomonas aeruginosa. Arch Intern Med 159:1127-1132

8. Cohen ML (1992) Epidemiology of drug resistance: implications for a post-antimicrobial era. Science 257:1050-1055

9. Holmberg SD, Solomon SL, Blake PA (1987) Health and economic impacts of antimicrobial resistance. Rev Infect Dis 9:1065-1078

10. Acar JF (1997) Consequences of bacterial resistance to antibiotics in medical practice. Clin Infect Dis 24 [Suppl 1]:S17-S18

11. Hartstein AI, Denny MA, Morthland VH, LeMonte AM, Pfaller MA (1995) Control of methicillin-resistant Staphylococcus aureus in a hospital and an intensive care unit. Infect Control Hosp Epidemiol 16:405-411

12. Klein BS, Perloff WH, Maki DG (1989) Reduction of nosocomial infection during pediatric intensive care by protective isolation. $\mathrm{N}$ Engl J Med 320:1714-1721

13. Slaughter S, Hayden MK, Nathan C, Hu TC, Rice T, Van Voorhis $\mathrm{J}$ et al (1996) A comparison of the effect of universal use of gloves and gowns with that of glove use alone on acquisition of 
vancomycin-resistant enterococci in a medical intensive care unit. Ann Intern Med 125:448-456

14. Bailey TC, Ritchie DJ, McMullin ST, Kahn M, Reichley RM, Casabar E et al (1997) A randomized, prospective evaluation of an interventional program to discontinue intravenous antibiotics at two tertiary care teaching institutions. Pharmacotherapy 17:277281

15. Pestotnik SL, Classen DC, Evans RS, Burke JP (1996) Implementing antibiotic practice guidelines through computer-assisted decision support: clinical and financial outcomes. Ann Intern Med 124:884-890

16. Quale J, Landman D, Saurina G, Atwood E, DiTore V, Patel K (1996) Manipulation of a hospital antimicrobial formulary to control an outbreak of vancomycin-resistant enterococci. Clin Infect Dis 23:1020-1025

17. Woodward RS, Medoff G, Smith MD, Gray JL 3rd (1987) Antibiotic cost savings from formulary restrictions and physician monitoring in a medical-school-affiliated hospital. Am J Med 83:817-823

18. Hughes MG, Evans HL, Chong TW, Smith RL, Raymond DP et al (2004) Effect of an intensive care unit rotating empiric antibiotic schedule on the development of hospital-acquired infections on the non-intensive care unit ward. Crit Care Med 32:53-60

19. Gruson D, Hilbert G, Vargas F, Valentino R, Bebear C, Allery A et al (2000) Rotation and restricted use of antibiotics in a medical intensive care unit. Impact on the incidence of ventilatorassociated pneumonia caused by antibiotic-resistant gramnegative bacteria. Am J Respir Crit Care Med 162:837-843

20. Gruson D, Hilbert G, Vargas F, Valentino R, Bui N, Pereyre S et al (2003) Strategy of antibiotic rotation: long-term effect on incidence and susceptibilities of Gram-negative bacilli responsible for ventilator-associated pneumonia. Crit Care Med 31:1908-1914

21. Raymond DP, Pelletier SJ, Crabtree TD, Gleason TG, Hamm LL, Pruett TL et al (2001) Impact of a rotating empiric antibiotic schedule on infectious mortality in an intensive care unit. Crit Care Med 29:1101-1108

22. Van Loon HJ, Vriens MR, Fluit AC, Troelstra A, van der Werken C, Verhoef J et al (2005) Antibiotic rotation and development of gram-negative antibiotic resistance. Am J Respir Crit Care Med 171:480-487

23. Gerding DN, Larson TA, Hughes RA, Weiler M, Shanholtzer C, Peterson LR (1991) Aminoglycoside resistance and aminoglycoside usage: ten years of experience in one hospital. Antimicrob Agents Chemother 35:1284-1290

24. Kollef MH, Vlasnik J, Sharpless L, Pasque C, Murphy D, Fraser V (1997) Scheduled change of antibiotic classes: a strategy to decrease the incidence of ventilator-associated pneumonia. Am J Respir Crit Care Med 156:1040-1048

25. Niederman MS (1997) Is "crop rotation" of antibiotics the solution to a "resistant" problem in the ICU? Am J Respir Crit Care Med 156:1029-1031

26. Warren DK, Hill HA, Merz LR, Kollef MH, Hayden MK, Fraser VJ et al (2004) Cycling empirical antimicrobial agents to prevent emergence of antimicrobial-resistant Gram-negative bacteria among intensive care unit patients. Crit Care Med $32: 2450-2456$

27. Dellit TH, Owens RC, McGowan JE, Gerding DN, Weinstein RA, Burke JP et al (2007) Infectious Diseases Society of America and the Society for Healthcare Epidemiology of America guidelines for developing an institutional program to enhance antimicrobial stewardship. Clin Infect Dis 44:159-177
28. Brown EM, Nathwani D (2005) Antibiotic cycling or rotation: a systematic review of the evidence of efficacy. J Antimicrob Chemother 55:6-9

29. Levy MM, Fink MP, Marshall JC, Abraham E, Angus D, Cook D et al (2003) $2001 \mathrm{SCCM} / \mathrm{ESICM} / \mathrm{ACCP} / \mathrm{ATS} / \mathrm{SIS}$ International Sepsis Definitions Conference. Crit Care Med 31:1250-1256

30. American Thoracic Society; Infectious Diseases Society of America (2005) Guidelines for the management of adults with hospital-acquired, ventilator-associated, and healthcare-associated pneumonia. Am J Respir Crit Care Med 171:388-416

31. Kollef MH, Bock KR, Richards RD, Hearns ML (1995) The safety and diagnostic accuracy of minibronchoalveolar lavage in patients with suspected ventilator-associated pneumonia. Ann Intern Med 122:743-748

32. Papazian L, Thomas P, Garbe L, Guignon I, Thirion X, Charrel J et al (1995) Bronchoscopic or blind sampling techniques for the diagnosis of ventilator-associated pneumonia. Am J Respir Crit Care Med 152:1982-1991

33. Calandra T, Cohen J (2005) The international sepsis forum consensus conference on definitions of infection in the intensive care unit. Crit CareMed 33:1538-1548

34. WHO Collaborating Centre for Drug Statistics Methodology (2006) ATC index with DDDs and guidelines for ATC classification and DDD assignment. Norwegian Institute of Public Health, Oslo

35. Pan A, Carnevale G, Catenazzi P et al (2005) Trends in methicillin-resistant Staphylococcus aureus (MRSA) bloodstream infections: effect of the MRSA "search and isolate" strategy in a hospital in Italy with hyperendemic MRSA. Infect Control Hosp Epidemiol 26:127-133

36. Raineri E, Crema L, De Silvestri A, Acquarolo A, Albertario F, Carnevale G et al (2007) Meticillin-resistant Staphylococcus aureus control in an intensive care unit: a 10 year analysis. J Hosp Infect 67:308-315

37. Wagner AK, Soumerai SB, Zhang F, Ross-Degnan D (2002) Segmented regression analysis of interrupted time series studies in medication use research. J Clin Pharm Ther 27:299-309

38. Bennett KM, Scarborough JE, Sharpe M, Dodds-Ashley E, Kaye KS, Hayward TZ 3rd et al (2007) Implementation of antibiotic rotation protocol improves antibiotic susceptibility profile in a surgical intensive care unit. J Trauma 63:307-311

39. Nijssen S, Bootsma M, Bonten M (2006) Potential confounding in evaluating infection-control interventions in hospital settings: changing antibiotic prescription. Clin Infect Dis 43(5):616-623

40. Fridkin SK (2003) Routine cycling of antimicrobial agents as an infection-control measure. Clin Infect Dis 36:1438-1444

41. Masterton RG (2005) Antibiotic cycling: more than it might seem? J Antimicrob Chemother 55:1-5

42. McGowan JE Jr (1987) Is antimicrobial resistance in hospital microorganisms related to antibiotic use? Bull N Y Acad Med $63: 253-268$

43. McGowan JE Jr, Gerding DN (1996) Does antibiotic restriction prevent resistance? New Horiz 4:370-376

44. Shlaes DM, Gerding DN, John JF Jr, Craig WA, Bornstein DL, Duncan RA et al (1997) Society for Healthcare Epidemiology of America and Infectious Diseases Society of America Joint Committee on the Prevention of Antimicrobial Resistance: guidelines for the prevention of antimicrobial resistance in hospitals. Clin Infect Dis 25:584-599

45. McGowan JE Jr (2000) Strategies for study of the role of cycling on antimicrobial use and resistance. Infect Control Hosp Epidemiol 21:S36-S43 